\title{
NUMBER OF X-RAY EXAMINATIONS PERFORMED ON PAEDIATRIC AND GERIATRIC PATIENTS COMPARED WITH ADULT PATIENTS
}

\author{
A. Aroua ${ }^{1, *}$, F. O. Bochud ${ }^{1}$, J.-F. Valley ${ }^{1}$, J.-P. Vader $^{2}$ and F. R. Verdun ${ }^{1}$ \\ ${ }^{1}$ University Institute of Radiation Physics (IRA-DUMSC), University of Lausanne, Grand-Pré 1, \\ CH-1007 Lausanne, Switzerland \\ ${ }^{2}$ University Institute of Social and Preventive Medicine (IUMSP-DUMSC), University of Lausanne, \\ Bugnon 17, CH-1005 Lausanne, Switzerland
}

Received April 20 2006, revised August 28 2006, accepted September 62006

\begin{abstract}
The age of the patient is of prime importance when assessing the radiological risk to patients due to medical $\mathrm{X}$-ray exposures and the total detriment to the population due to radiodiagnostics. In order to take into account the age-specific radiosensitivity, three age groups are considered: children, adults and the elderly. In this work, the relative number of examinations carried out on paediatric and geriatric patients is established, compared with adult patients, for radiodiagnostics as a whole, for dental and medical radiology, for 8 radiological modalities as well as for 40 types of X-ray examinations. The relative numbers of X-ray examinations are determined based on the corresponding age distributions of patients and that of the general population. Two broad groups of X-ray examinations may be defined. Group A comprises conventional radiography, fluoroscopy and computed tomography; for this group a paediatric patient undergoes half the number of examinations as that of an adult, and a geriatric patient undergoes 2.5 times more. Group B comprises angiography and interventional procedures; for this group a paediatric patient undergoes a one-fourth of the number of examinations carried out on an adult, and a geriatric patient undergoes five times more.
\end{abstract}

\section{INTRODUCTION}

The age of the patient is quite an important parameter for the evaluation of the detriment of medical $\mathrm{X}$-ray exposures. The risks associated with an exposure for elderly patients (induction of cancer with 20 y latency, hereditary disorders in the offspring) are less important than those for adult patients, whereas children are more radiosensitive than adults.

To take into account the effect of age in estimating the radiological risk, various models are proposed in literature to correct the effective dose, such as the BfS model ${ }^{(1)}$ or the NRPB model ${ }^{(2)}$. They all suggest the use of a multiplying factor decreasing with age to weight the effective dose. Usually three age categories are used: children, adults and the elderly with boundaries that may change slightly from one model to another.

It is, therefore, important, when assessing the impact of diagnostic and interventional radiology on the population, to establish the age distribution of patients undergoing X-ray examinations and to determine the fraction of these examinations associated with paediatric, adult and geriatric patients.

This work aims at determining the number of X-ray examinations performed on children and the elderly, considering adults as a reference, for a

*Corresponding author: abbas@aroua.com number of radiological modalities and a series of common radiological examinations.

\section{METHODS}

In this work the following age groups are adopted:

(1) Paediatric patients: below age 15.

(2) Adult patients: between age 15 and age 64 .

(3) Geriatric patients: age 65 and above.

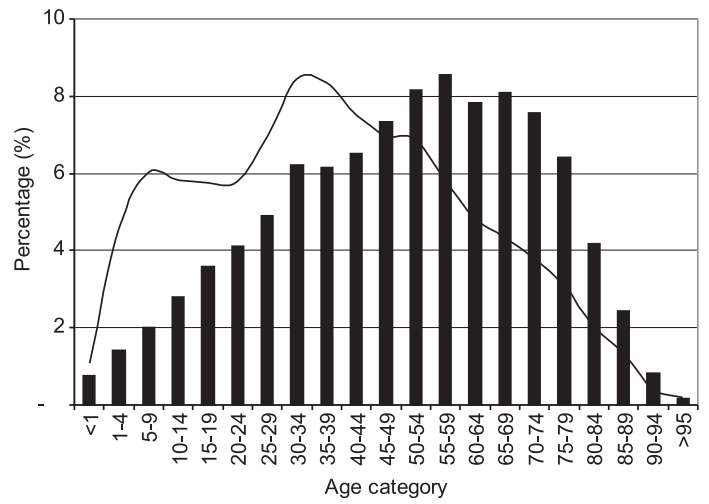

Figure 1. Patient age profile (histograms) related to all radiological modalities (average age of 50). The age profile of the Swiss general population is shown in solid line; with a corresponding average age of 39. 
X-RAY EXAMINATIONS IN PAEDIATRICS AND GERIATRICS

Table 1. Number of X-ray examinations performed on paediatric and geriatric patients relative to adult patients for various radiological modalities.

\begin{tabular}{|c|c|c|c|c|c|c|c|c|}
\hline \multirow[t]{2}{*}{ Radiological modality } & \multirow{2}{*}{\multicolumn{2}{|c|}{$A$}} & \multicolumn{5}{|c|}{ Age group } & \multirow[t]{2}{*}{ All ages } \\
\hline & & & $(0-14)$ & $(15-39)$ & $(40-64)$ & $(15-64)$ & $(65+)$ & \\
\hline Population & 39 & $N$ & $1,242,329$ & $2,507,375$ & $2,270,978$ & $4,778,353$ & $1,076,212$ & $7,096,894$ \\
\hline Tedi & 50 & $N$ & 666,713 & $2,376,014$ & $3,660,720$ & $6,036,734$ & $2,826,757$ & $9,530,204$ \\
\hline & & $\begin{array}{l}N_{\mathrm{a}} \\
N_{\mathrm{p}} \\
R\end{array}$ & $\begin{array}{c}7 \\
537 \\
0.42\end{array}$ & $\begin{array}{r}25 \\
948 \\
-\end{array}$ & $\begin{array}{r}38 \\
1,612 \\
-\end{array}$ & $\begin{array}{c}63 \\
1,263 \\
1.00\end{array}$ & $\begin{array}{c}30 \\
2,627 \\
2.08\end{array}$ & $\begin{array}{r}100 \\
1,343\end{array}$ \\
\hline Dental X-ray & 42 & $N$ & 269,532 & $1,630,979$ & $1,724,134$ & $3,355,113$ & 498,833 & $4,123,478$ \\
\hline & & $\begin{array}{l}N_{\mathrm{a}} \\
N_{\mathrm{p}} \\
R\end{array}$ & $\begin{array}{c}7 \\
217 \\
0.31\end{array}$ & $\begin{array}{r}40 \\
650 \\
-\end{array}$ & $\begin{array}{r}41 \\
759 \\
-\end{array}$ & $\begin{array}{c}81 \\
702 \\
1.00\end{array}$ & $\begin{array}{c}12 \\
464 \\
0.66\end{array}$ & $\begin{array}{r}100 \\
581 \\
\end{array}$ \\
\hline $\begin{array}{l}\text { Medical X-ray } \\
\text { examinations }\end{array}$ & 56 & $N$ & 397,207 & 744,212 & $1,936,394$ & $2,680,606$ & $2,328,913$ & $5,406,726$ \\
\hline & & $\begin{array}{l}N_{\mathrm{a}} \\
N_{\mathrm{p}} \\
R\end{array}$ & $\begin{array}{c}7 \\
320 \\
0.57\end{array}$ & $\begin{array}{r}14 \\
297 \\
-\end{array}$ & $\begin{array}{r}36 \\
853 \\
-\end{array}$ & $\begin{array}{c}50 \\
561 \\
1.00\end{array}$ & $\begin{array}{c}43 \\
2,164 \\
3.86\end{array}$ & $\begin{array}{r}100 \\
762 \\
-\end{array}$ \\
\hline Radiography & 48 & $\begin{array}{l}N \\
N_{\mathrm{a}} \\
N_{\mathrm{p}} \\
R\end{array}$ & $\begin{array}{c}466,160 \\
10 \\
375 \\
0.66\end{array}$ & $\begin{array}{r}1,206,473 \\
27 \\
481 \\
-\end{array}$ & $\begin{array}{r}1,511,371 \\
33 \\
666 \\
-\end{array}$ & $\begin{array}{r}2,717,844 \\
60 \\
569 \\
1.00\end{array}$ & $\begin{array}{r}1,375,460 \\
30 \\
1,278 \\
2.25\end{array}$ & $\begin{array}{r}4,559,465 \\
100 \\
642 \\
-\end{array}$ \\
\hline $\begin{array}{l}\text { Conventional } \\
\text { luoroscopy }\end{array}$ & 48 & $N$ & 20,713 & 30,954 & 57,928 & 88,882 & 46,205 & 155,799 \\
\hline & & $\begin{array}{l}N_{\mathrm{a}} \\
N_{\mathrm{p}} \\
R\end{array}$ & $\begin{array}{l}13 \\
17 \\
0.90\end{array}$ & $\begin{array}{l}20 \\
12 \\
-\end{array}$ & $\begin{array}{l}37 \\
26 \\
-\end{array}$ & $\begin{array}{l}57 \\
19 \\
1.00\end{array}$ & $\begin{array}{l}30 \\
43 \\
2.31\end{array}$ & $\begin{array}{r}100 \\
22 \\
-\end{array}$ \\
\hline onventional & 52 & $N$ & - & 2,931 & 5,326 & 8,257 & 3,107 & 11,364 \\
\hline & & $\begin{array}{l}N_{\mathrm{a}} \\
N_{\mathrm{p}} \\
R\end{array}$ & - & $\begin{array}{l}26 \\
1.2 \\
-\end{array}$ & $\begin{array}{l}47 \\
2.3 \\
-\end{array}$ & $\begin{array}{l}73 \\
1.7 \\
1.00\end{array}$ & $\begin{array}{l}27 \\
2.9 \\
1.67\end{array}$ & $\begin{array}{c}100 \\
1.6 \\
-\end{array}$ \\
\hline Computed tomography & 54 & $\begin{array}{l}N \\
N_{\mathrm{a}} \\
N_{\mathrm{p}} \\
R\end{array}$ & $\begin{array}{r}6,703 \\
2 \\
5 \\
0.12\end{array}$ & $\begin{array}{r}71,339 \\
22 \\
28 \\
\end{array}$ & $\begin{array}{r}139,124 \\
42 \\
61 \\
-\end{array}$ & $\begin{array}{r}210,463 \\
64 \\
44 \\
1.00\end{array}$ & $\begin{array}{r}111,318 \\
34 \\
103 \\
2.35\end{array}$ & $\begin{array}{r}328,484 \\
100 \\
46 \\
-\end{array}$ \\
\hline Mammography & 55 & $\begin{array}{l}N \\
N_{\mathrm{a}} \\
N_{\mathrm{p}} \\
R\end{array}$ & $\begin{array}{l}63 \\
0 \\
0.05 \\
0.00\end{array}$ & $\begin{array}{r}19,120 \\
9 \\
8 \\
-\end{array}$ & $\begin{array}{r}158,840 \\
71 \\
70 \\
-\end{array}$ & $\begin{array}{r}177,960 \\
80 \\
37 \\
1.00\end{array}$ & $\begin{array}{r}44,554 \\
20 \\
41 \\
1.11\end{array}$ & $\begin{array}{r}222,577 \\
100 \\
31 \\
-\end{array}$ \\
\hline Angiography & 60 & $\begin{array}{l}N \\
N_{\mathrm{a}} \\
N_{\mathrm{p}} \\
R\end{array}$ & $\begin{array}{c}2,930 \\
4 \\
2.4 \\
0.33\end{array}$ & $\begin{array}{r}5,374 \\
8 \\
2.1 \\
-\end{array}$ & $\begin{array}{r}28,742 \\
41 \\
13 \\
-\end{array}$ & $\begin{array}{c}34,116 \\
49 \\
7.1 \\
1.00\end{array}$ & $\begin{array}{r}33,024 \\
47 \\
31 \\
4.30\end{array}$ & $\begin{array}{r}70,070 \\
100 \\
10 \\
-\end{array}$ \\
\hline Bone densitometry & 61 & $\begin{array}{l}N \\
N_{\mathrm{a}} \\
N_{\mathrm{p}} \\
R\end{array}$ & $\begin{array}{l}272 \\
1 \\
0.22 \\
0.05\end{array}$ & $\begin{array}{l}376 \\
1 \\
0.15 \\
-\end{array}$ & $\begin{array}{c}18,999 \\
59 \\
8.4 \\
-\end{array}$ & $\begin{array}{c}19,375 \\
60 \\
4.1 \\
1.00\end{array}$ & $\begin{array}{c}12,573 \\
39 \\
12 \\
2.88\end{array}$ & $\begin{array}{c}32,220 \\
100 \\
4.5 \\
-\end{array}$ \\
\hline Interventional procedures & 63 & $\begin{array}{l}N \\
N_{\mathrm{a}} \\
N_{\mathrm{p}} \\
R\end{array}$ & $\begin{array}{l}534 \\
2 \\
0.4 \\
0.18\end{array}$ & $\begin{array}{c}1,711 \\
6 \\
0.7 \\
-\end{array}$ & $\begin{array}{c}9,768 \\
37 \\
4.3 \\
-\end{array}$ & $\begin{array}{c}11,479 \\
43 \\
2.4 \\
1.00\end{array}$ & $\begin{array}{r}14,734 \\
55 \\
14 \\
5.70\end{array}$ & $\begin{array}{c}26,747 \\
100 \\
3.8 \\
-\end{array}$ \\
\hline
\end{tabular}

$A$ : mean value of the age distribution

$N$ : number of examinations performed annually in Switzerland (or Swiss population) within the corresponding age group $N_{\mathrm{a}}$ : fraction (\%) of the number of examinations for the corresponding age group

$N_{\mathrm{p}}$ : number of examinations per 1000 population of the corresponding age group;

$R: N_{\mathrm{p}}$ for the corresponding age group/ $N_{\mathrm{p}}(15-64)$. 


\section{A. AROUA ET AL}

Table 2. Number of X-ray examinations performed on paediatric and geriatric patients relative to adult patients for two broad groups of X-ray examinations.

\begin{tabular}{lccc}
\hline $\begin{array}{l}\text { Group of X-ray } \\
\text { examinations }\end{array}$ & $\begin{array}{c}\text { Paediatric } \\
\text { patients } \\
(0-14)\end{array}$ & $\begin{array}{c}\text { Adult } \\
\text { patients } \\
(15-64)\end{array}$ & $\begin{array}{c}\text { Geriatric } \\
\text { patients } \\
(65+)\end{array}$ \\
\hline $\begin{array}{l}\text { Group A } \\
\text { Radiography, } \\
\quad \begin{array}{l}\text { Conventional } \\
\text { fluoroscopy, Computed } \\
\text { tomography }\end{array}\end{array}$ & 0.5 & 1 & 2.5 \\
$\begin{array}{l}\text { Group B } \\
\text { Angiography, }\end{array}$ & & & \\
$\quad$ Interventional radiology & 0.25 & 1 & 5 \\
\hline
\end{tabular}

In this study patients above 65 are considered as geriatric patients. The United Nations Scientific Committee on the Effects of the Atomic Radiation (UNSCEAR) $^{(3)}$ provides age data in different age groups: $0-15,16-40$ and $>40$. In order to provide data in the present work that can be used in the UNSCEAR format, the group of adult patients (15-64) is divided into two subgroups (15-39 and 40-64). The second subgroup added to the geriatric group leads to the third UNSCEAR group.

If $n_{\mathrm{pa}}(a)$ and $n_{\mathrm{gp}}(a)$ represent the age distribution of patients who undergo X-ray examinations and that of the general population, respectively, the fractions of paediatric, adult and geriatric patients may then be expressed, respectively, as follows:

$$
\int_{0}^{15} n_{\mathrm{pa}}(a) \mathrm{d} a, \quad \int_{15}^{65} n_{\mathrm{pa}}(a) \mathrm{d} a, \quad \int_{65}^{\infty} n_{\mathrm{pa}}(a) \mathrm{d} a .
$$

Similarly, the fractions of children, adults and the elderly within the general population may be expressed, respectively, as follows:

$$
\int_{0}^{15} n_{\mathrm{gp}}(a) \mathrm{d} a, \quad \int_{15}^{65} n_{\mathrm{gp}}(a) \mathrm{d} a, \quad \int_{65}^{\infty} n_{\mathrm{gp}}(a) \mathrm{d} a .
$$

If the adult patients are taken as a reference, the relative number of examinations for paediatric patients, $R_{\text {ped }}$, may be written as follows:

$$
R_{\mathrm{ped}}=\frac{\int_{0}^{15} n_{\mathrm{pa}}(a) \mathrm{d} a / \int_{0}^{15} n_{\mathrm{gp}}(a) \mathrm{d} a}{\int_{15}^{65} n_{\mathrm{pa}}(a) \mathrm{d} a / \int_{15}^{65} n_{\mathrm{gp}}(a) \mathrm{d} a} .
$$

Similarly, the relative number of examinations for geriatric patients, $R_{\mathrm{ger}}$, may be written as follows:

$$
R_{\mathrm{ger}}=\frac{\int_{65}^{\infty} n_{\mathrm{pa}}(a) \mathrm{d} a / \int_{65}^{\infty} n_{\mathrm{gp}}(a) \mathrm{d} a}{\int_{15}^{65} n_{\mathrm{pa}}(a) \mathrm{d} a / \int_{15}^{65} n_{\mathrm{gp}}(a) \mathrm{d} a} .
$$

The age distributions used in this work, $n_{\mathrm{pa}}(a)$, are established from the data collected during the 1998 nationwide survey on the exposure of the Swiss population by radiodiagnostics ${ }^{(4-6)}$, while the age distribution of the Swiss general population, $n_{\mathrm{gp}}(a)$, for the same year, is established using the official published data ${ }^{(7)}$.

Figure 1 shows the age distribution $n_{\mathrm{pa}}(a)$ for the total number of X-ray examinations carried out in Switzerland considering all the radiological modalities and compares it with the age distribution of the Swiss general population.

The number of X-ray examinations performed on paediatric and geriatric patients, relative to adult patients, is determined for eight radiological modalities: (1) radiography (except mammography), (2) conventional fluoroscopy (non-vascular, used mainly in gastro-enterology, urology and genecology), (3) computed tomography, (4) angiography, (5) interventional radiology, (6) mammography, (7) conventional tomography, and (8) bone densitometry, as well as for 40 types of examinations covering these eight radiological modalities.

\section{RESULTS AND DISCUSSION}

Table 1 presents the number of X-ray examinations for paediatric and geriatric patients, relative to adult patients, $R$, first for the whole radiological profession, then separately for dental and medical radiology and last for eight medical radiological modalities. These modalities are sorted by ascending average age $(A)$. In each case, the annual total number of examinations $(N)$, the fraction of that number $\left(N_{\mathrm{a}}\right.$ in $\left.\%\right)$ for each age group and the number of examination per 1000 population $\left(N_{\mathrm{p}}\right)$ are given as well.

Table 1 indicates that if all medical and dental examinations are considered, then a geriatric patient undergoes twice the number of X-ray examinations as that of an adult patient, whereas a paediatric patient undergoes less than half the number as that of an adult.

When splitting the total number of X-ray examinations into dental and medical, two significantly different distributions are obtained with average ages of 42 and 56, respectively. In the case of dental examinations, geriatric patients undergo on average two-thirds the number of dental examinations as that of adult patients whereas paediatric patients undergo less than one-third. As regards medical examinations, the relative number of examinations for geriatric patients is $\sim 4$ and that for paediatric patients is $\sim 0.6$.

If the eight medical radiological modalities are looked at separately, one finds that except for mammography where a geriatric patient has only $10 \%$ more examinations than an adult patient, in all other modalities the relative number of examinations in geriatrics is high and ranges between 1.7 for conventional tomography and 5.7 for interventional radiology. Concerning paediatrics, the relative 


\section{X-RAY EXAMINATIONS IN PAEDIATRICS AND GERIATRICS}

Table 3. Number of $X$-ray examinations performed on paediatric and geriatric patients relative to adult patients for a series of 40 types of examinations.

\begin{tabular}{|c|c|c|c|c|c|c|c|c|}
\hline \multirow[t]{2}{*}{$\mathrm{X}$-ray examination } & \multirow[t]{2}{*}{$A$} & & \multicolumn{5}{|c|}{ Age group } & \multirow[t]{2}{*}{ All ages } \\
\hline & & & $(0-14)$ & $(15-39)$ & $(40-64)$ & $(15-64)$ & $(65+)$ & \\
\hline Population & 39 & $N$ & $1,242,329$ & $2,507,375$ & $2,270,978$ & $4,778,353$ & $1,076,212$ & $7,096,894$ \\
\hline \multirow{4}{*}{$\begin{array}{l}\text { Micturating cysto- } \\
\text { urethrography (MCU) }\end{array}$} & 11 & $N$ & 9,583 & 597 & 540 & 1,137 & 617 & 11,337 \\
\hline & & $N_{\mathrm{a}}$ & 85 & 5 & 5 & 10 & 5 & 100 \\
\hline & & $N_{\mathrm{p}}$ & 7.7 & 0.24 & 0.24 & 0.24 & 0.57 & 1.6 \\
\hline & & $R$ & 32.42 & - & - & 1.00 & 2.41 & - \\
\hline \multirow[t]{4}{*}{ Skull radiography } & 35 & $N$ & 20,263 & 35,407 & 21,308 & 56,715 & 13,467 & 90,445 \\
\hline & & $N_{\mathrm{a}}$ & 22 & 39 & 24 & 63 & 15 & 100 \\
\hline & & $N_{\mathrm{p}}$ & 16 & 14 & 9 & 12 & 13 & 13 \\
\hline & & $R$ & 1.37 & - & - & 1.00 & 1.05 & - \\
\hline \multirow[t]{4}{*}{ Hand radiography } & 36 & $N$ & 32,637 & 48,591 & 37,301 & 85,892 & 20,728 & 139,257 \\
\hline & & $N_{\mathrm{a}}$ & 23 & 35 & 27 & 62 & 15 & 100 \\
\hline & & $N_{\mathrm{p}}$ & 26 & 19 & 16 & 18 & 19 & 20 \\
\hline & & $R$ & 1.46 & - & - & 1.00 & 1.07 & - \\
\hline \multirow[t]{4}{*}{ Bitewing radiography } & 37 & $N$ & 115,132 & 847,054 & 640,935 & $1,487,989$ & 91,326 & $1,694,447$ \\
\hline & & $N_{\mathrm{a}}$ & 7 & 50 & 38 & 88 & 5 & 100 \\
\hline & & $N_{\mathrm{p}}$ & 93 & 338 & 282 & 311 & 85 & 239 \\
\hline & & $R$ & 0.30 & - & - & 1.00 & 0.27 & - \\
\hline \multirow[t]{4}{*}{ Orthopantomography (OPG) } & 41 & $N$ & 21,712 & 93,330 & 87,982 & 181,312 & 33,638 & 236,662 \\
\hline & & $N_{\mathrm{a}}$ & 9 & 40 & 37 & 77 & 14 & 100 \\
\hline & & $N_{\mathrm{p}}$ & 17 & 37 & 39 & 38 & 31 & 33 \\
\hline & & $R$ & 0.46 & - & - & 1.00 & 0.82 & - \\
\hline \multirow[t]{4}{*}{ Cervical spine radiography } & 43 & $N$ & 6,944 & 62,080 & 61,173 & 123,253 & 22,233 & 152,430 \\
\hline & & $N_{\mathrm{a}}$ & 5 & 41 & 40 & 81 & 15 & 100 \\
\hline & & $N_{\mathrm{p}}$ & 6 & 25 & 27 & 26 & 21 & 21 \\
\hline & & $R$ & 0.22 & - & - & 1.00 & 0.80 & - \\
\hline \multirow[t]{4}{*}{ Foot radiography } & 44 & $N$ & 20,764 & 59,028 & 60,691 & 119,719 & 36,468 & 176,951 \\
\hline & & $N_{\mathrm{a}}$ & 12 & 33 & 34 & 67 & 21 & 100 \\
\hline & & $N_{\mathrm{p}}$ & 17 & 24 & 27 & 25 & 34 & 25 \\
\hline & & $R$ & 0.67 & - & - & 1.00 & 1.35 & - \\
\hline \multirow[t]{4}{*}{ Cerebral embolisation } & 44 & $N$ & 18 & 200 & 233 & 433 & 68 & 519 \\
\hline & & $N_{\mathrm{a}}$ & 4 & 38 & 45 & 83 & 13 & 100 \\
\hline & & $N_{\mathrm{p}}$ & 0.01 & 0.08 & 0.10 & 0.09 & 0.06 & 0.07 \\
\hline & & $\mathrm{R}^{\mathrm{p}}$ & 0.16 & - & - & 1.00 & 0.69 & - \\
\hline \multirow[t]{4}{*}{ Thoracic spine radiography } & 46 & $N$ & 6,717 & 24,518 & 24,757 & 49,275 & 18,644 & 74,637 \\
\hline & & $N_{\mathrm{a}}$ & 9 & 33 & 33 & 66 & 25 & 100 \\
\hline & & $N_{\mathrm{p}}$ & 5 & 10 & 11 & 10 & 17 & 11 \\
\hline & & $R$ & 0.52 & - & - & 1.00 & 1.68 & - \\
\hline \multirow[t]{4}{*}{ Apical radiography } & 47 & $N$ & 85,591 & 619,628 & 949,679 & $1,569,307$ & 363,763 & $2,018,661$ \\
\hline & & $N_{\mathrm{a}}$ & 4 & 31 & 47 & 78 & 18 & 100 \\
\hline & & $N_{\mathrm{p}}$ & 69 & 247 & 418 & 328 & 338 & 284 \\
\hline & & $R$ & 0.21 & - & & 1.00 & 1.03 & - \\
\hline \multirow[t]{4}{*}{ Intravenous urography (UIV) } & 48 & $N$ & 1,421 & 10,054 & 14,104 & 24,158 & 7,027 & 32,607 \\
\hline & & $N_{\mathrm{a}}$ & 4 & 31 & 43 & 74 & 22 & 100 \\
\hline & & $N_{\mathrm{p}}$ & 1.1 & 4.0 & 6.2 & 5.1 & 6.5 & 4.6 \\
\hline & & $R$ & 0.23 & - & - & 1.00 & 1.29 & - \\
\hline \multirow[t]{4}{*}{ Knee radiography } & 48 & $N$ & 21,921 & 103,423 & 106,521 & 209,944 & 96,028 & 327,893 \\
\hline & & $N_{\mathrm{a}}$ & 7 & 32 & 32 & 64 & 29 & 100 \\
\hline & & $N_{\mathrm{p}}$ & 18 & 41 & 47 & 44 & 89 & 46 \\
\hline & & $R$ & 0.40 & - & - & 1.00 & 2.03 & - \\
\hline Abdomen radiography & 49 & $N$ & 11,485 & 41,815 & 48,051 & 89,866 & 42,846 & 144,197 \\
\hline & & $N_{\mathrm{a}}$ & 8 & 29 & 33 & 62 & 30 & 100 \\
\hline & & $N_{\mathrm{p}}$ & 9 & 17 & 21 & 19 & 40 & 20 \\
\hline & & $R$ & 0.49 & - & - & 1.00 & 2.12 & - \\
\hline Barium meal & 49 & $N$ & 2,111 & 2,212 & 5,028 & 7,240 & 4,459 & 13,810 \\
\hline & & $N_{\mathrm{a}}$ & 15 & 16 & 36 & 52 & 32 & 100 \\
\hline
\end{tabular}




\begin{tabular}{|c|c|c|c|c|c|c|c|c|}
\hline \multirow{2}{*}{ X-ray examination } & \multirow[t]{2}{*}{$A$} & & \multicolumn{5}{|c|}{ Age group } & \multirow[t]{2}{*}{ All ages } \\
\hline & & & $(0-14)$ & $(15-39)$ & $(40-64)$ & $(15-64)$ & $(65+)$ & \\
\hline & & $N_{\mathrm{p}}$ & 1.7 & 0.9 & 2.2 & 1.5 & 4.1 & 1.9 \\
\hline & & $R$ & 1.12 & - & - & 1.00 & 2.73 & - \\
\hline \multirow{4}{*}{ Pelvis CT } & 49 & $N$ & 186 & 1,932 & 2,395 & 4,327 & 1,587 & 6,100 \\
\hline & & $N_{\mathrm{a}}$ & 3 & 32 & 39 & 71 & 26 & 100 \\
\hline & & $N_{\mathrm{p}}$ & 0.15 & 0.8 & 1.0 & 0.9 & 1.5 & 0.9 \\
\hline & & $R$ & 0.17 & - & - & 1.00 & 1.63 & \\
\hline \multirow{4}{*}{ Lumbar spine radiography } & 50 & $N$ & 9,254 & 79,492 & 111,130 & 190,622 & 73,091 & 272,967 \\
\hline & & $N_{\mathrm{a}}$ & 3 & & & 70 & 27 & \\
\hline & & $N_{\mathrm{p}}$ & 7 & 32 & 49 & 40 & 68 & 38 \\
\hline & & $R$ & 0.19 & - & - & 1.00 & 1.70 & - \\
\hline \multirow{4}{*}{ Lumbar spine $\mathrm{CT}$} & 52 & $N$ & 134 & 9,485 & 18,583 & 28,068 & 9,542 & 37,744 \\
\hline & & $N_{\mathrm{a}}$ & 0 & 25 & & 74 & 25 & \\
\hline & & $N_{\mathrm{p}}$ & 0.11 & 3.8 & 8.2 & 5.9 & 8.9 & 5.3 \\
\hline & & $R$ & 0.02 & - & - & 1.00 & 1.51 & - \\
\hline \multirow[t]{4}{*}{ Skull CT } & 54 & $N$ & 2,457 & 18,272 & 31,314 & 49,586 & 26,946 & 78,988 \\
\hline & & $N_{\mathrm{a}}$ & 3 & & & 63 & 34 & 100 \\
\hline & & $N_{\mathrm{p}}^{a}$ & 2.0 & 7.3 & 14 & 10 & 25 & 11 \\
\hline & & $R$ & 0.19 & - & - & 1.00 & 2.41 & - \\
\hline \multirow[t]{4}{*}{ Ascending pyelography } & 54 & $N$ & 399 & 2,091 & 3,839 & 5,930 & 3,827 & 10,155 \\
\hline & & $N_{\mathrm{a}}$ & 4 & 20 & 38 & 58 & 38 & \\
\hline & & $N_{\mathrm{p}}$ & 0.3 & 0.8 & 1.7 & 1.2 & 3.6 & 1.4 \\
\hline & & $R$ & 0.26 & - & - & 1.00 & 2.87 & - \\
\hline \multirow[t]{4}{*}{ Chest radiography } & 54 & $N$ & 126,040 & 226,768 & 536,620 & 763,388 & 599,528 & $1,488,956$ \\
\hline & & $N_{\mathrm{a}}$ & 8 & 15 & 36 & 51 & 40 & \\
\hline & & $N_{\mathrm{p}}$ & 101 & 90 & 236 & 160 & 557 & 210 \\
\hline & & $R$ & 0.64 & - & & 1.00 & 3.49 & - \\
\hline \multirow{4}{*}{ Shoulder radiography } & 54 & $N$ & 5,367 & 42,661 & 60,786 & 103,447 & 63,439 & 172,253 \\
\hline & & $N_{\mathrm{a}}$ & 3 & 25 & 35 & 60 & 37 & 100 \\
\hline & & $N_{\mathrm{p}}$ & 4 & 17 & 27 & 22 & 59 & 24 \\
\hline & & $R$ & 0.20 & - & - & 1.00 & 2.72 & - \\
\hline \multirow{4}{*}{ Chest CT } & 54 & $N$ & 1,251 & 9,227 & 21,159 & 30,386 & 16,044 & 47,682 \\
\hline & & $N_{\mathrm{a}}$ & 3 & 19 & & 63 & 34 & \\
\hline & & $N_{\mathrm{p}}$ & 1.0 & 3.7 & 9.3 & 6.4 & 15 & 6.7 \\
\hline & & $R$ & 0.16 & - & - & 1.00 & 2.34 & - \\
\hline \multirow[t]{4}{*}{ Mammography } & 55 & $N$ & 63 & 19,120 & 158,840 & 177,960 & 44,554 & 222,577 \\
\hline & & $N_{\mathrm{a}}$ & 0 & 9 & 71 & 80 & 20 & \\
\hline & & $N_{\mathrm{p}}$ & 0.1 & 7.6 & 70 & 37 & 41 & 31 \\
\hline & & $R$ & 0.00 & - & - & 1.00 & 1.11 & - \\
\hline \multirow[t]{4}{*}{ Pelvis radiography } & 56 & $N$ & 18,659 & 43,172 & 69,564 & 112,736 & 109,520 & 240,914 \\
\hline & & $N_{\mathrm{a}}$ & 8 & 18 & 29 & 47 & 45 & 100 \\
\hline & & $N_{\mathrm{p}}$ & 15 & 17 & 31 & 24 & 102 & 34 \\
\hline & & $R$ & 0.64 & - & - & 1.00 & 4.31 & - \\
\hline \multirow[t]{4}{*}{ Abdomen CT } & 58 & $N$ & 800 & 16,729 & 40,543 & 57,272 & 38,890 & 96,962 \\
\hline & & $N_{\mathrm{a}}$ & 1 & 17 & & 59 & 40 & 100 \\
\hline & & $N_{\mathrm{p}}$ & 0.6 & 6.7 & 18 & 12 & 36 & 14 \\
\hline & & $R$ & 0.05 & - & - & 1.00 & 3.01 & - \\
\hline \multirow[t]{4}{*}{ Barium enema } & 58 & $\mathrm{~N}$ & 724 & 466 & 2,649 & 3,115 & 3,374 & 7,213 \\
\hline & & $N_{\mathrm{a}}$ & 10 & 6 & 37 & 43 & 47 & 100 \\
\hline & & $N_{\mathrm{p}}$ & 0.6 & 0.2 & 1.2 & 0.7 & 3.1 & 1.0 \\
\hline & & $R$ & 0.89 & - & - & 1.00 & 4.81 & - \\
\hline Pelvic angiography & 60 & $N$ & 28 & 243 & 1,105 & 1,348 & 1,135 & 2,511 \\
\hline & & $N_{\mathrm{a}}$ & 1 & 10 & 44 & 54 & 45 & 100 \\
\hline & & $N_{-}^{a}$ & 0.02 & 0.10 & 0.49 & 0.28 & 1.0 & 0.35 \\
\hline & & $R$ & 0.08 & - & - & 1.00 & 3.74 & - \\
\hline Retrograde chol- & 60 & $N$ & 30 & 799 & 1,902 & 2,701 & 2,507 & 5,238 \\
\hline & & $N_{\mathrm{a}}$ & 1 & 15 & 36 & 51 & 48 & 100 \\
\hline
\end{tabular}


Table 3. Continued

\begin{tabular}{|c|c|c|c|c|c|c|c|c|}
\hline \multirow[t]{2}{*}{ X-ray examination } & \multirow{2}{*}{\multicolumn{2}{|c|}{$A$}} & \multicolumn{5}{|c|}{ Age group } & \multirow[t]{2}{*}{ All ages } \\
\hline & & & $(0-14)$ & $(15-39)$ & $(40-64)$ & $(15-64)$ & $(65+)$ & \\
\hline & & $N_{\mathrm{p}}$ & 0.02 & 0.32 & 0.84 & 0.57 & 2.3 & 0.74 \\
\hline & & $R$ & 0.04 & - & - & 1.00 & 4.12 & - \\
\hline \multirow[t]{4}{*}{ Biliary drainage } & 61 & $N$ & 30 & 90 & 311 & 401 & 436 & 867 \\
\hline & & $N_{\mathrm{a}}$ & 3 & 10 & 36 & 46 & 50 & 100 \\
\hline & & $N_{\mathrm{p}}$ & 0.02 & 0.04 & 0.14 & 0.08 & 0.41 & 0.12 \\
\hline & & $R$ & 0.28 & - & - & 1.00 & 4.83 & - \\
\hline \multirow[t]{4}{*}{ Abdominal embolisation } & 61 & $N$ & 8 & 26 & 89 & 115 & 125 & 248 \\
\hline & & $N_{\mathrm{a}}$ & 3 & 10 & 36 & 46 & 50 & 100 \\
\hline & & $N_{\mathrm{p}}^{\mathrm{a}}$ & 0.01 & 0.01 & 0.04 & 0.02 & 0.12 & 0.03 \\
\hline & & $R$ & 0.28 & - & - & 1.00 & 4.83 & - \\
\hline \multirow[t]{4}{*}{ Chest angiography } & 61 & $N$ & - & 200 & 541 & 741 & 690 & 1,431 \\
\hline & & $N_{\mathrm{a}}$ & - & 14 & 38 & 52 & 48 & 100 \\
\hline & & $N_{\mathrm{p}}$ & - & 0.08 & 0.24 & 0.15 & 0.64 & 0.20 \\
\hline & & $R$ & - & - & - & 1.00 & 4.13 & - \\
\hline \multirow[t]{4}{*}{ Coronary angiography } & 63 & $N$ & 33 & 730 & 9,038 & 9,768 & 9,414 & 19,215 \\
\hline & & $N_{\mathrm{a}}$ & 0 & 4 & 47 & 51 & 49 & 100 \\
\hline & & $N_{\mathrm{p}}$ & 0.03 & 0.29 & 4.0 & 2.0 & 8.7 & 2.7 \\
\hline & & $R$ & 0.01 & - & - & 1.00 & 4.28 & - \\
\hline \multirow[t]{4}{*}{ Coronary dilatation (PTCA) } & 63 & $N$ & 29 & 245 & 4,615 & 4,860 & 4,803 & 9,692 \\
\hline & & $N_{\mathrm{a}}$ & 0 & 2 & 48 & 50 & 50 & 100 \\
\hline & & $N_{\mathrm{p}}$ & 0.02 & 0.10 & 2.0 & 1.0 & 4.5 & 1.4 \\
\hline & & $R$ & 0.02 & - & - & 1.00 & 4.39 & - \\
\hline \multirow[t]{4}{*}{ Hip radiography } & 64 & $N$ & 7,035 & 9,554 & 22,839 & 32,393 & 61,135 & 100,562 \\
\hline & & $N_{\mathrm{a}}$ & 7 & 9 & 23 & 32 & 61 & 100 \\
\hline & & $N_{\mathrm{p}}$ & 5.7 & 3.8 & 10 & 6.8 & 57 & 14 \\
\hline & & $R$ & 0.84 & - & - & 1.00 & 8.38 & - \\
\hline \multirow[t]{4}{*}{ Cerebral angiography } & 64 & $N$ & - & 93 & 835 & 928 & 1,100 & 2,028 \\
\hline & & $N_{\mathrm{a}}$ & - & 5 & 41 & 46 & 54 & 100 \\
\hline & & $N_{\mathrm{p}}$ & - & 0.04 & 0.37 & 0.19 & 1.02 & 0.29 \\
\hline & & $R$ & - & - & - & 1.00 & 5.26 & - \\
\hline \multirow[t]{4}{*}{ Upper limb angiography } & 65 & $N$ & 3 & 78 & 310 & 389 & 563 & 954 \\
\hline & & $N_{\mathrm{a}}$ & 0 & 8 & 33 & 41 & 59 & 100 \\
\hline & & $N_{\mathrm{p}}$ & 0.002 & 0.03 & 0.14 & 0.08 & 0.52 & 0.13 \\
\hline & & $R$ & 0.03 & - & - & 1.00 & 6.43 & - \\
\hline \multirow[t]{4}{*}{ Abdominal angiography } & 66 & $N$ & 23 & 243 & 1,878 & 2,121 & 3,354 & 5,498 \\
\hline & & $N_{\mathrm{a}}$ & 0 & 5 & 34 & 39 & 61 & 100 \\
\hline & & $N_{\mathrm{p}}$ & 0.02 & 0.10 & 0.83 & 0.44 & 3.1 & 0.77 \\
\hline & & $R$ & 0.04 & - & - & 1.00 & 7.02 & - \\
\hline \multirow{4}{*}{ Lower limb angiography } & 69 & $N$ & 11 & 152 & 1,329 & 1,481 & 3,287 & 4,780 \\
\hline & & $N_{\mathrm{a}}$ & 0 & 3 & 28 & 31 & 69 & 100 \\
\hline & & $N_{\mathrm{p}}$ & 0.01 & 0.06 & 0.59 & 0.31 & 3.0 & 0.67 \\
\hline & & $R$ & 0.03 & - & - & 1.00 & 9.85 & - \\
\hline \multirow[t]{4}{*}{ Femoral dilatation } & 71 & $N$ & 3 & 19 & 664 & 683 & 1,823 & 2,509 \\
\hline & & $N_{\mathrm{a}}$ & 0 & 1 & 26 & 27 & 73 & 100 \\
\hline & & $N_{\mathrm{p}}$ & 0.003 & 0.008 & 0.29 & 0.14 & 1.7 & 0.35 \\
\hline & & $R$ & 0.02 & - & - & 1.00 & 11.85 & - \\
\hline \multirow[t]{4}{*}{ Pacemaker insertion } & 74 & $N$ & 52 & 125 & 235 & 360 & 2,785 & 3,197 \\
\hline & & $N_{\mathrm{a}}$ & 2 & 4 & 7 & 11 & 87 & 100 \\
\hline & & $N_{\mathrm{p}}$ & 0.04 & 0.05 & 0.10 & 0.07 & 2.6 & 0.45 \\
\hline & & $R$ & 0.55 & - & - & 1.00 & 34.28 & - \\
\hline
\end{tabular}

$A$ : mean value of the age distribution

$N$ : number of examinations performed annually in Switzerland (or Swiss population) within the corresponding age group $N_{\mathrm{a}}$ : fraction (\%) of the number of examinations for the corresponding age group $N_{\mathrm{p}}$ : number of examinations per 1000 population of the corresponding age group $R: N_{\mathrm{p}}$ for the corresponding age group/ $N_{\mathrm{p}}(15-64)$. 


\section{A. AROUA ET AL.}

number varies between 0 or almost 0 for some modalities such as mammography, conventional tomography and bone densitometry and 0.9 for conventional fluoroscopy, where children seem to be almost equally exposed to X-ray examinations as adults.

From Table 1 it is possible to define two broad groups of common $\mathrm{X}$-ray examinations with simple rounded values of the relative numbers of examinations for paediatric and geriatric patients, as shown in Table 2. For group A (radiography, conventional fluoroscopy and computed tomography), a paediatric patient undergoes half the number of examinations as that of an adult one, while a geriatric patient undergoes 2.5 times more. For group B (angiography, interventional radiology), the differences are doubled, since a paediatric patient undergoes one-fourth the number of examinations as that of an adult one, while a geriatric patient undergoes five times more.

Table 3 presents the same information given in Table 1 for 40 types of X-ray examinations (dental and medical, diagnostic and interventional). The types of examinations are sorted by ascending average age.

The series shown in Table 3 extends from what can be considered as mostly paediatric examinations such as micturating cysto-urethrography, a ratio of $30: 1$ paediatrics/adults in this study, to what can be seen as mostly geriatric examinations such as pacemaker insertion (more than 30 examinations on the elderly for one examination on adults). In most cases, paediatric patients undergo fewer X-ray examinations than adult patients who themselves undergo fewer X-ray examinations than geriatric patients, except for a limited number of cases. For four types of examinations $(10 \%$ of the total), micturating cysto-urethrography, skull radiography, hand radiography and barium meal, paediatric patients undergo more X-ray examinations than adult patients, whereas for four other types of examinations $(10 \%$ of the total), bitewing radiography, orthopantomography (OPG), cervical spine radiography and cerebral embolisation, geriatric patients undergo fewer X-ray examinations than adult patients.

\section{CONCLUSION}

The relative number of $\mathrm{X}$-ray examinations performed on paediatric and geriatric patients, compared with adult patients, was established using patient age distributions and that of the general population. This number was determined for 8 radiological modalities and 40 types of X-ray examinations covering medical and dental radiology. It was found that in most cases an adult patient undergoes more examinations than a paediatric patient and less than a geriatric patient. Two broad groups of examinations may be defined. The first one includes conventional radiography, fluoroscopy and computed tomography, while the second comprises angiography and interventional radiology. For the first group an adult undergoes twice as many examinations as a child and 2.5 times less than an elderly patient. For the second group, consisting of dose-intensive examinations, the difference is even larger, since an adult undergoes four times more examinations than a child and one-fifth the number of examinations performed on an elderly patient.

\section{REFERENCES}

1. Bernhardt, J. H., Veit, R. and Bauer, B. Radiation exposure of the German population from $X$-ray diagnostic procedures. In: Proceedings of the Ninth Congress of the International Radiation Protection Association 3, Vienna, pp. 383-385 (1996).

2. Shrimpton, P. C., Wall, B. F., Croft, J. R. and Webb, G. A. M. Medical Exposure: Guidance on the 1990 Recommendations of ICRP. In: Documents of the NRPB: Occupational, Public and Medical Exposure 4(2), Didcot (1993).

3. United Nations Scientific Committee on the Effects of Atomic Radiation. 2000 Report to the General Assembly, Annex D: medical radiation exposures. (NY: UN) (2000).

4. Aroua, A., Burnand, B., Decka, I., Vader, J. P. and Valley, J.-F. Nation-wide survey on radiation doses in diagnostic and interventional radiology in Switzerland in 1998. Health Phys. 83(1), 46-55 (2002).

5. Aroua, A., Burnand, B., Decka, I., Vader, J. P. and Valley, J.-F. Dosimetric aspects of a national survey of diagnostic and interventional radiology in Switzerland. Med. Phys. 29(10), 2247-2259 (2002).

6. Aroua, A., Vader, J.-P. and Valley, J.-F. A survey on exposure by diagnostic and interventional radiology in Switzerland in 1998. Institut Universitaire de Radiophysique Appliquée, Lausanne. Available on: www. hospvd.ch/public/instituts/ira. (2000).

7. Service cantonal de recherche et d'information statistique. Annuaire statistique du Canton de Vaud, Lausanne (1999). 